PROCEEDINGS OF THE

AMERICAN MATHEMATICAL SOCIETY

Volume 131, Number 9, Pages 2921-2932

S 0002-9939(03)07120-X

Article electronically published on April 23, 2003

\title{
CONVEXITY OF MOMENT POLYTOPES OF ALGEBRAIC VARIETIES
}

\author{
WILLIAM LIU \\ (Communicated by Mohan Ramachandran)
}

\begin{abstract}
We consider the situation of a compact irreducible subvariety of a smooth compact complex variety equipped with a Kähler form preserved by a torus action. We study the image of that subvariety under the moment map of the Kähler form.
\end{abstract}

\section{Main Result}

The moment map of a Hamiltonian $T$-symplectic/Kähler manifold has been one of the main interests of study for mathematical as well as physical reasons. In the early 80's, Atiyah in [A] considered the following situation:

Let $M$ be a compact finite dimensional Kähler manifold on which a real torus acts in a Hamiltonian fashion. There is then a natural extension of the real torus action to the complexified torus action by applying the almost complex structure induced by the complex structure to the infinitesimal real torus action and then integrating to obtain the action of the "imaginary part" of the complexified torus.

He proved the following:

Theorem $1.1([\mathrm{~A}])$. Let $f$ be a moment map of $M$ with respect to the torus action. Let $Y$ be an orbit of the complexified torus, and let $Z_{1}, \cdots, Z_{p}$ be the components of the set of all fixed points of the torus lying in the closure of $Y$. Then the image of the closure of $Y$ under the moment map is equal to the convex hull of the images of $c_{1}, \cdots, c_{p}$ where $c_{i}=f\left(Z_{i}\right)$ for each $i$.

The convex hull in the theorem is sometimes called the moment polytope.

One can ask if the above theorem holds for more general subspaces than a single orbit of $M$. The answer turns out to be positive in the following setting:

Suppose that $M$ is a compact finite dimensional smooth complex variety equipped with a Kähler form $\omega$. Let $T$ be a real torus acting on $(M, \omega)$ that preserves $\omega$ and the complex structure such that the moment map exists. Denote its Lie algebra by t. Let $T_{\mathbb{C}}$ be the complexified torus which also acts on $M$. Let $X$ be a compact

Received by the editors May 25, 2001.

2000 Mathematics Subject Classification. Primary 53D20, 37J15, 58E40; Secondary 22E67, $14 \mathrm{M} 15$.

Key words and phrases. Symplectic structures, Hamiltonian torus action, moment maps, Morse theory, loop groups. 
irreducible subvariety, possibly singular, of $M$ invariant under the $T_{\mathbb{C}}$ action. We then have:

Theorem 1.2. Let $\mu: M \longrightarrow \mathbf{t}^{*}$ be the moment map of $\omega$. Then the image of $X$ under $\mu$ coincides with the convex hull of the images of all $T_{\mathbb{C}}$-fixed points in $X$ under $\mu$.

Remarks 1.3. (1) A simple but important observation is that a point in $M$ fixed by $T$ is also fixed by $T_{\mathbb{C}}$ and vice versa. This fact applies to all the spaces we shall consider in this paper.

(2) Using a bi-invariant inner product on $\mathbf{t}$, we shall freely identify $\mathbf{t}$ with its dual $\mathbf{t}^{*}$.

Proof of Theorem 1.2. We shall prove that

(a) the image of $X$ is contained in the convex hull, and

(b) the image contains the convex hull.

Proof of (a). To prove part (a), we need the following two simple and well-known lemmas:

Lemma 1.4. Let $D \subset \mathbb{R}^{n}, \theta_{1}, \cdots, \theta_{p} \in D$. If for any $v \in \mathbb{R}^{n}$, the image of $D$ under the orthogonal projection (denoted by $\mu$ ) on the line $\mathbb{R} \cdot v$ equals an interval of the form $\left[\mu\left(\theta_{i}\right), \mu\left(\theta_{j}\right)\right]$ for some $i, j$, then $D$ is contained in the convex hull of $\theta_{1}, \cdots, \theta_{p}$.

Proof. Suppose there exists an element $x$ in $D$ which is not in the convex hull of $\theta_{1}, \cdots, \theta_{p}$. Then $x$ must be on the opposite side to the convex hull of a hyperplane defined by a face with vertices, say, $\theta_{1}, \cdots, \theta_{k}$. Choose a vector $v \in \mathbb{R}^{n}$ which is perpendicular to that face. Then one can easily see that the image of $x$ under the projection on the straight line $\mathbb{R} v$ is outside $\left[\mu\left(\theta_{i}\right), \mu\left(\theta_{j}\right)\right]$ for any $i, j$, which leads to a contradiction.

Remark 1.5. Notice that the above lemma still holds if "for any $v$ " is replaced by "for almost all $v$ ".

Lemma 1.6. Let $G$ be a compact Lie group and $(M, \omega, G)$ a Hamiltonian $G$-space with moment map $\mu: M \rightarrow \mathbf{g}$ where $\mathbf{g}$ is the Lie algebra of $G$. Let $H$ be a subgroup of $G$ which is not necessarily closed and $\mathbf{h}$ its Lie algebra. Then $(M, \omega, H)$ is a Hamiltonian $H$-space with moment map given by the composite

$$
M \stackrel{\mu}{\longrightarrow} \mathbf{g} \stackrel{p r}{\longrightarrow} \mathbf{h}
$$

where $p r$ is the orthogonal projection with respect to the bi-invariant metric on $G$ used to identify $\mathbf{g}$ and $\mathbf{g}^{*}$.

We can now carry out the proof of part (a). Regarding $\mathbf{t}$ as a Euclidean space, we show that for any $\alpha \in \mathbf{t}$ with irrational coordinates (so that the subgroup $\exp (\mathbb{R} \cdot \alpha)$ is dense in $T)$, the image of $\mu(X)$ under the orthogonal projection on the line $\mathbb{R} \cdot \alpha$ equals $\left[\mu\left(\theta_{i}\right), \mu\left(\theta_{j}\right)\right]$ for some fixed points $\theta_{i}, \theta_{j}$ in $X$. Consider the composite map

$$
\tilde{\mu}: X \stackrel{\mu}{\longrightarrow} \mathbf{t} \longrightarrow \mathbb{R} \cdot \alpha
$$

where the second map is the orthogonal projection. Since $X$ is compact connected, its image under $\tilde{\mu}$ is a closed interval. The maximum and minimum of that interval will be images of some critical points of $\tilde{\mu}$. Notice that $\mathbb{R} \cdot \alpha$ is just the Lie algebra 
of the subgroup $\exp (\mathbb{R} \cdot \alpha)$ of $T$. Therefore by Lemma 1.6, $\tilde{\mu}$ is the restriction of the moment map with respect to that subgroup. Hence the critical points of this map are the same as the fixed points of the subgroup, which are precisely the fixed points of the whole torus $T$ because the subgroup is dense in $T$. The desired result now follows from Lemma 1.4.

Proof of (b). Following an idea used in Atiyah-Pressley $\underline{\mathrm{A}-\mathrm{P}}$, one could first try to show that there exists an element $y$ in $X$ such that the closure of the complexified torus orbit $T_{\mathbb{C}} \cdot y$ contains all fixed points of $T_{\mathbb{C}}$ in $X$ and then apply Theorem 1.1 However, there may be some fixed points $x \in X$ such that $\mu(x)$ is not a vertex of the convex hull. We shall prove a weaker statement that there exists $y$ in $X$ such that the image of the closure of $T_{\mathbb{C}} \cdot y$ under $\mu$ contains all vertices of the convex hull of the images of all fixed points of $T_{\mathbb{C}}$ in $X$ under $\mu$, and then apply Theorem 1.1 This weaker statement is sufficient for proving part (b).

Let $c_{1}, \ldots, c_{p}$ be the vertices of the convex hull of the image of all $T$-fixed points in $X$ under $\mu$. For each $c_{i}$, take a hyperplane which touches the convex hull only at $c_{i}$. Let $v_{i}^{*}$ be the unit vector normal to the hyperplane and points into the convex hull, and let $L_{i}=\mathbb{R} v_{i}^{*}$ be the vector subspace spanned by $v_{i}^{*}$. Consider the composite

$$
f_{i}: M \stackrel{\mu}{\longrightarrow} \mathbf{t}^{*} \stackrel{\pi_{i}}{\longrightarrow} L_{i} \stackrel{s_{i}}{\longrightarrow} \mathbb{R}
$$

where $\pi_{i}$ is the orthogonal projection onto $L_{i}$ and $s_{i}$ is a linear map sending $v_{i}^{*}$ to $1 \in \mathbb{R}$, which is an isomorphism. For technical reasons, we choose $v_{i}^{*}$ such that its dual vector $v_{i} \in \mathbf{t}$ generates a dense subgroup $G=\left\{\exp \left(t v_{i}\right) \in T \mid t \in \mathbb{R}\right\}$ of $T$. Then $f_{i}$ is just the moment map of the action of $G$ on $M$. Notice that $G$ is isomorphic to $\mathbb{R}$, and we have:

Lemma 1.7. This action extends to a $\mathbb{C}^{*}$ action on $M$.

There is a well-known result:

Lemma $1.8([\mathrm{~A}],[\mathrm{F}])$. The function $f_{i}$ is Morse-Bott.

This lemma can be proved by a straightforward calculation in local normal coordinates.

Now, notice that $s_{i} \circ \pi_{i}\left(c_{i}\right)$ is the absolute minimal value of $\left.f_{i}\right|_{X}$. Let

$$
F_{i}^{1}, \ldots, F_{i}^{k} \subset M
$$

be all the critical submanifolds that intersect $X$. Consider the stable manifolds

$$
M_{i}^{s}=\left\{m \in M \mid m \text { flows down to } F_{i}^{s} \text { along some gradient flow line of } f_{i}\right\}
$$

for $s=1, \cdots, k$. Here a gradient flow line is a smooth curve $x(t)$ on $M$ satisfying the differential equation

$$
\frac{d}{d t} x(t)=-\left.\operatorname{grad} f_{i}\right|_{x(t)} .
$$

The condition defining $M_{i}^{s}$ means that $m$ lies on some gradient flow line $x(t)$ and

$$
\lim _{x \rightarrow \infty} x(t) \in F_{i}^{s}
$$

Recall that in Lemma 1.7, we defined a $\mathbb{C}^{*}$ action on $M$. We have

Lemma 1.9. Gradient flow lines of $f_{i}$ are generated by this $\mathbb{C}^{*}$ action. 
Proof. Fix any point $m \in M$. Let $x(t), t \in \mathbb{R}$, be the gradient flow line such that $x(0)=m$. Recall that in the definition of $f_{i}$, there is a vector $v_{i}^{*} \in \mathbf{t}^{*}$ and its dual $v_{i} \in \mathbf{t}$ which generates $G$. Then $\sqrt{-1} v_{i}$ is in the complexification $\mathbb{C}^{*} \subset \mathbf{t}_{\mathbb{C}}$. Consider the curve $a(t)=\exp \left(t \sqrt{-1} v_{i}\right) m$ on $M$. Notice that $\exp \left(\sqrt{-1} v_{i}\right)$ is a curve on $\mathbb{C}^{*}$. Hence $a(t)$ is generated by the $\mathbb{C}^{*}$ action. We claim that it coincides with $x(t)$. For any $t$, write $x=a(t)$, and for any $X \in T_{x} M$, we have

$$
\begin{aligned}
\left\langle X, \frac{d}{d t} a(t)\right\rangle_{M} & =\left\langle X, J\left(v_{i} \cdot x\right)\right\rangle_{M} \\
& =\omega\left(X, J^{2}\left(v_{i} \cdot x\right)\right) \\
& =-\omega\left(X, v_{i} \cdot x\right) \\
& =-\left\langle d \mu(X), v_{i}\right\rangle \\
& =-\pi_{i} \circ d \mu(X) \\
& =\left\langle X,-\operatorname{grad} f_{i}\right\rangle_{M}
\end{aligned}
$$

where $\langle\rangle:, \mathbf{t}^{*} \times \mathbf{t} \rightarrow \mathbb{R}$ is the canonical pairing. Hence we have

$$
\frac{d}{d t} a(t)=-\operatorname{grad} f_{i}
$$

that is, $a(t)=x(t)$.

Therefore, we have an alternative description of $M_{i}^{s}$ :

$$
M_{i}^{s}=\left\{m \in M \mid \lim _{t \rightarrow \infty} \exp \left(t \sqrt{-1} v_{i}\right) \cdot m \in F_{i}^{s}\right\}
$$

for $s=1, \cdots, k$. By a result of [C-S] $(\S \mathrm{Ic}), \overline{M_{i}^{s}}$ is a subvariety of $M$, and $M_{i}^{s}$ is Zariski open in $\overline{M_{i}^{s}}$ for each $s$.

Every point of $X$ must flow down to some critical submanifold. Hence $X$ is contained in the union $\bigcup_{s=1}^{k} M_{i}^{s}$.

Lemma 1.10. The set $X$ is contained in $\overline{M_{i}^{s}}$ for some $s$.

Proof. Observe that

$$
X \subset \bigcup_{s=1}^{k} M_{i}^{s} \subset \bigcup_{s=1}^{k} \overline{M_{i}^{s}} .
$$

Therefore $X=\bigcup_{s=1}^{k}\left(X \cap \overline{M_{i}^{s}}\right)$.

Since $\overline{M_{i}^{s}}$ is a subvariety of $M$, the intersection $X \cap \overline{M_{i}^{s}}$ is a subvariety of $X$ for $s=1, \cdots, k$. By the irreducibility of $X$, we must have

$$
X=X \cap \overline{M_{i}^{s}}
$$

for some $s$. This implies that $X \subset \overline{M_{i}^{s}}$.

Lemma 1.11. $f_{i}\left(F_{i}^{s}\right)=c_{i}$.

Proof. Since $c_{i}$ is the absolute minimal value of $\left.f_{i}\right|_{X}, f_{i}\left(F_{i}^{s}\right) \geq c_{i}$. Notice that the restriction of $f_{i}$ to a gradient flow line $a(t)$ must be decreasing with respect to $t$. Moreover, by Morse theory, the closure of a stable manifold of a critical submanifold $F$ precisely consists of points which are initial points of (downward) broken gradient flow lines that go down to $F$. A (downward) broken gradient flow line is a piecewise smooth curve such that each smooth segment is a gradient flow line. 
By these two facts, we can see that the value of each point $x \in \overline{M_{i}^{s}}$ under $f_{i}$ must be greater than or equal to $f_{i}\left(F_{i}^{s}\right)$. And since $c_{i} \in f_{i}(X)$, we must have $c_{i} \geq f_{i}\left(F_{i}^{s}\right)$.

Since $M_{i}^{s}$ is Zariski open in $\overline{M_{i}^{s}}$ and $X \cap M_{i}^{s} \neq \emptyset, X \cap M_{i}^{s}$ must be Zariski open in $X$. Therefore the finite intersection

$$
\bigcap_{i=1}^{k} X \cap M_{i}^{s_{i}}
$$

is also Zariski open in $X$. In particular it is non-empty. Take any element $y$ in that intersection and consider the complex torus orbit $T_{\mathbb{C}} \cdot y$.

Lemma 1.12. The image of the closure of the orbit $T_{\mathbb{C}} \cdot y$ under $\mu$ contains all vertices $c_{1}, \ldots, c_{p}$ of the convex hull.

Proof. For each $i$, since $y \in X \cap M_{i} \subset M_{i}$, there is a flow line $x(t)$ with respect to $f_{i}$ that contains $y$ and $x(t)$ flows down to $F_{i}^{s}$ for some $s$. But we saw that gradient flow lines can be obtained by the $\mathbb{C}^{*}$ action. In particular, $x(t)$ is entirely contained in the orbit $T_{\mathbb{C}} \cdot y$. Hence the image of the closure of $T_{\mathbb{C}} \cdot y$ under $\mu$ contains $c_{i}$. This completes the proof of the lemma.

By Theorem 1.1, the image of the closure of $T_{\mathbb{C}} \cdot y$ under $\mu$ is equal to the convex hull of the images of all $T$-fixed points in $T_{\mathbb{C}} \cdot y$. Hence by the above lemma, it contains the convex hull of all $c_{i}$, and we have

$$
\mu(X) \supset \mu\left(\text { the closure of } T_{\mathbb{C}} \cdot y\right) \supset \text { convex hull }\left\langle c_{1}, \ldots, c_{p}\right\rangle,
$$

which concludes the proof of part (b) of the theorem.

Hence the proof of Theorem 1.2 is complete.

Remark 1.13. The irreducibility condition of the subvariety $X$ in Theorem 1.2 is necessary. Consider the following example:

Let $M=\mathbb{C} P^{2}$ be equipped with the standard Kähler structure. Let $T=\mathbf{S}^{1} \times \mathbf{S}^{1}$ act on $M$ by

$$
(a, b) \cdot\left[z_{0} ; z_{1} ; z_{2}\right]=\left[z_{0} ; a z_{1} ; b z_{2}\right] .
$$

This torus action defines a moment map $\mu: M \rightarrow \mathbb{R}^{2}$.

Let $X=\left\{\left[z_{0} ; z_{1} ; z_{2}\right] \in \mathbb{C} P^{2} \mid z_{1} z_{2}=0\right\}$. It is a compact, reducible algebraic subvariety on $\mathbb{C} P^{2}$. By simple calculation, one can see that $\mu(X)$ is a union of two line segments meeting at one point, whereas there are three fixed points in $X$ : $[1 ; 0 ; 0],[0 ; 1 ; 0]$ and $[0 ; 0 ; 1]$ such that the convex hull of their images under $\mu$ is a triangle.

\section{Applichtions}

2.1. Convexity theorem. In [A] and [G-S], a theorem, which is now usually referred to as the convexity theorem in symplectic geometry, is proved:

Theorem 2.1 (Convexity Theorem $[\mathrm{A}], \mathrm{G}-\mathrm{S}]$ ). Let $(M, \omega)$ be a finite dimensional symplectic manifold with a real torus $T$ acting on $M$ that preserves $\omega$ such that a moment map $\mu$ exists. Then the image of $M$ under the moment map is equal to the convex hull of the images of all T-fixed points under $\mu$. 
In [A], it is suggested that one could use the idea of Theorem[1.1 to prove a Kähler version of Theorem 2.1 We shall now realize this suggestion by using the idea in the proof of Theorem [1.2 to prove the following Kähler version of Theorem [2.1;

Theorem 2.2. Let $(M, \omega, J)$ be a compact Kähler manifold where $\omega$ is the Kähler (symplectic) form invariant under the complex structure $J$. Let $T$ be a real torus acting on $M$ which preserves $\omega$ and $J$ such that a moment map $\mu$ exists. Then the image of $M$ under $\mu$ is equal to the convex hull of the images of all $T$-fixed points in $M$.

Proof. We show that

(a) the image of $M$ is contained in the convex hull, and

(b) the image contains the convex hull.

The proof of part (a) is again the same as in Theorem 1.2. For part (b), let $c_{1}, \cdots, c_{p}$ be the vertices of the convex hull. For each $i$, construct the Morse-Bott function

$$
f_{i}: M \stackrel{\mu}{\longrightarrow} \mathbf{t}^{*} \stackrel{\pi_{i}}{\longrightarrow} L_{i} \stackrel{s_{i}}{\longrightarrow} \mathbb{R}
$$

as in the proof of Theorem[1.2 Let $m_{1}^{i}, \ldots, m_{k}^{i} \in M$ be the preimages of $c_{i}$ under $\mu$. They are the absolute minima of $f_{i}$. Let $M_{i}$ be the union of the stable manifolds of the $m_{j}^{i}, j=1, \ldots, k$. Notice that these stable manifolds are open and dense in $M$. Hence the finite intersection $\bigcap_{i=1}^{p} M_{i}$ is also open and dense in $M$. In particular, it is non-empty. Take an element $y$ in $\bigcap_{i=1}^{p} M_{i}$. Then as in the proof of Lemma 1.12 the image of the closure of the orbit $T_{\mathbb{C}} \cdot y$ under $\mu$ contains all vertices $c_{1}, \ldots, c_{p}$ of the convex hull. Hence we have

$$
\mu(X) \supset \mu\left(\text { the closure of } T_{\mathbb{C}} \cdot y\right) \supset \text { convex hull }\left\langle c_{1}, \ldots, c_{p}\right\rangle
$$

where the second inclusion follows from Theorem 1.1. This concludes the proof of part (b).

2.2. Loop groups. In this section, we shall generalize Theorem 1.2 to an infinite dimensional situation, replacing $M$ by an "infinite dimensional subvariety" (which will be defined later) of the loop group $\Omega U_{n}$ which is the set of all smooth functions (called "loops") from the unit circle $\mathbf{S}^{1} \subset \mathbb{C}$ into the unitary group $U_{n}$ which send 1 to the identity matrix.

We first recall briefly various structures and properties of $\Omega U_{n}$ relevant to this paper, following $\mathrm{A}-\mathrm{P}]$. For details, see $[\mathrm{L}]$ or $[\mathrm{P}-\mathrm{S}]$.

With pointwise multiplication, $\Omega U_{n}$ is a group. In fact, it is a Lie group, modeled on the infinite dimensional Lie algebra $\Omega u_{n}$ which is the set of all smooth maps from $\mathbf{S}^{1}$ to the Lie algebra $u_{n}$ of $U_{n}$ which send 1 to $0 \in u_{n}$.

1. There is a left invariant complex structure and a left invariant symplectic structure on $\Omega U_{n}$ defined as follows:

For any $u(s) \in \Omega u_{n}$, consider the Laurent expansion:

$$
u(s)=\sum_{k \neq 0} A_{k} e^{i k s},
$$

where each $A_{k}$ is a complex $n \times n$ matrix. Define

$$
J u(s)=\sum_{k<0} i A_{k} e^{i k s}-\sum_{k>0} i A_{k} e^{i k s} .
$$


It is easy to see that this gives a linear map $J: \Omega u_{n} \rightarrow \Omega u_{n}$ such that $J^{2}=-1$. By left translation using the group structure of $\Omega U_{n}$, we have an almost complex structure $J$ on $\Omega U_{n}$.

For any two tangent vectors $X(\lambda), Y(\lambda)$ in the Lie algebra $\Omega u_{n}$, set

$$
\omega_{A P}(X, Y)=\frac{1}{2 \pi} \int_{\mathbf{S}^{1}}\left\langle X(s), Y^{\prime}(s)\right\rangle d s
$$

where $\langle\cdot, \cdot\rangle$ is the bi-invariant inner product on $u_{n}$ defined by

$$
\langle A, B\rangle=\operatorname{Tr}\left(A^{*} B\right) .
$$

Here $\lambda=e^{i s}$ is the loop parameter. By left translation again, we get a left invariant 2 -form $\omega_{A P}$ on $\Omega U_{n}$.

It turns out that $J$ is integrable, and that $\omega_{A P}$ is a symplectic form such that $\left(\Omega U_{n}, \omega_{A P}, J\right)$ becomes a Kähler manifold.

2. There is an action of a real torus $T \times \mathbf{S}^{1}$ on $\Omega U_{n}$, where $T$ is the maximal torus consisting of diagonal matrices in $U_{n}$, defined as follows: $T$ acts on $U_{n}$ by conjugation, which induces an action on $\Omega U_{n}$; and $\mathbf{S}^{1}$ action on $\Omega U_{n}$ by "rotating the loops". More precisely, for any $z \in \mathbf{S}^{1}$ and $\gamma(\lambda) \in \Omega U_{n}$, the action is defined to be

$$
z \cdot \gamma(\lambda)=\gamma(z \cdot \lambda) \gamma(z)^{-1}
$$

It is not hard to see that this torus action preserves $J$ and $\omega_{A P}$. It also extends to an action of the complexified torus $\left(T \times \mathbf{S}^{1}\right)_{\mathbb{C}}=T_{\mathbb{C}} \times \mathbb{C}^{*}$.

3. A moment map exists, which can be defined explicitly as follows:

For any $f(s)$ in $\Omega U_{n}$, define the energy function

$$
E: \Omega U_{n} \rightarrow \mathbb{R}: f \mapsto \frac{1}{4 \pi} \int_{\mathbf{S}^{1}}\left\|f^{\prime}(s) f(s)^{-1}\right\|^{2} d s
$$

and the momentum function

$$
p: \Omega U_{n} \rightarrow \mathbf{t}: f \mapsto p r_{\mathbf{t}} \frac{1}{2 \pi} \int_{\mathbf{S}^{1}} f^{\prime}(s) f(s)^{-1} d s
$$

where $p r_{\mathbf{t}}$ is the orthogonal projection to $\mathbf{t}$. Then the map

$$
\mu_{A P}=p \oplus E: \Omega U_{n} \longrightarrow \mathbf{t} \oplus \mathbb{R}
$$

is a moment map for the action of $T \times \mathbf{S}^{1}$ on $\Omega U_{n}$.

4. Consider the set of "algebraic loops" in $U_{n}$ :

$\Omega_{\text {alg }} U_{n}=\left\{\gamma \in \Omega U_{n} \mid \gamma(\lambda)=\sum_{i=-N}^{N} A_{i} \lambda^{i}\right.$ for some $N \in \mathbf{Z}$ and $n \times n$ matrices $\left.A_{i}\right\}$.

It is a subgroup of $\Omega U_{n}$. Moreover, it has a structure of an "infinite dimensional" algebraic variety defined as follows:

First, consider its identity component $\left(\Omega_{\text {alg }} U_{n}\right)_{0}=\Omega_{a l g} S U_{n}$. Let

$$
M_{k}=\left\{\gamma \in \Omega_{\text {alg }} U_{n} \mid \gamma \text { is a polynomial in } \lambda^{-1}, \operatorname{deg} \operatorname{det} \gamma=-k\right\} .
$$

Observe that there is a filtration

$$
M_{0} \subset \lambda M_{n} \subset \lambda^{2} M_{2 n} \subset \cdots \subset \bigcup_{k \geq 0} \lambda^{k} M_{k n}=\Omega_{a l g} S U_{n}
$$

where

$$
\lambda M_{n}=\left\{\hat{\gamma} \in \Omega_{a l g} U_{n} \mid \hat{\gamma}(\lambda)=\lambda \cdot \gamma(\lambda) \text { for some } \gamma \in M_{n}\right\},
$$

etc. This filtration is called the Mitchell-Segal filtration $\underline{\mathrm{M}}, \mathbf{S}$. 
Each space $M_{k n}$, as well as $\lambda^{k} M_{k n}$, has a structure of finite dimensional algebraic variety that we now define. Consider the Hilbert subspace of the space of all $\mathrm{L}^{2}$ functions from $\mathbf{S}^{1}$ to $\mathbb{C}^{n}$ :

$$
H_{+}=\overline{\operatorname{Span}\left\{\lambda^{i} e_{j}: i \geq 0, j=1, \ldots, n\right\}} \subset L^{2}\left(\mathbf{S}^{1} ; \mathbb{C}^{n}\right)
$$

where $\left\{e_{1}, \ldots, e_{n}\right\}$ is the standard basis of $\mathbb{C}^{n}$ and the closure is taken with respect to the $L^{2}$-norm. Notice that for any $\gamma \in M_{k n}$, since it is a polynomial in $\lambda^{-1}, \gamma^{*}$ is a polynomial in $\lambda$. So $\gamma^{*} H_{+} \subset H_{+}$. This implies that $H_{+} \subset \gamma H_{+}$. On the other hand, since $\operatorname{deg} \operatorname{det} \gamma=-k n$, we have $\lambda^{k n} \gamma \in H_{+}$, which implies $\gamma H_{+} \subset \lambda^{-k n} H_{+}$. In sum, we have

$$
H_{+} \subset \gamma H_{+} \subset \lambda^{-k n} H_{+} .
$$

It can be shown that, since deg det $\gamma=-k n, \gamma H_{+}$has codimension $k n$ in $\lambda^{-k} H_{+}$. (See, for example, [P-S].) Equivalently, the quotient space $\left(\gamma H_{+}\right) / H_{+}$is a $k n$ dimensional vector subspace of $\lambda^{-k n} H_{+} / H_{+}$. Hence there is a map

$$
\varphi: M_{k n} \rightarrow G r_{k n}\left(\mathbb{C}^{k n^{2}}\right): \gamma \mapsto\left(\gamma H_{+}\right) / H_{+} \subset \lambda^{-k n} H_{+} / H_{+} .
$$

Here we identify $\lambda^{-k n} H_{+} / H_{+} \cong\left\langle\lambda^{i} e_{j}: j=1, \cdots, n ; i=-1, \cdots,-k n\right\rangle$ with $\mathbb{C}^{k n^{2}}$.

It is easy to see that $\varphi$ is an injection under which the image of $M_{k n}$ is a compact irreducible algebraic subvariety of the Grassmannian.

As for $\lambda^{k} M_{k n}$, notice that for any $\gamma \in M_{k n}$, we have

$$
\lambda^{k} H_{+} \subset \lambda^{k} \gamma H_{+} \subset \lambda^{k-k n} H_{+} .
$$

Similar to above, the quotient $\left(\lambda^{k} \gamma H_{+}\right) /\left(\lambda^{k} H_{+}\right)$is a $k n$-dimensional vector subspace of

$$
\lambda^{k-k n} H_{+} /\left(\lambda^{k} H_{+}\right) \cong\left\langle\lambda^{i} e_{j}: j=1, \cdots, n ; i=k-k n, \cdots, k-1\right\rangle \cong \mathbb{C}^{k n^{2}},
$$

and that this defines an embedding of $\lambda^{k} M_{k n}$ into a Grassmannian of $k n$-planes in $\mathbb{C}^{k n^{2}}$ as a compact irreducible algebraic subvariety. We shall denote this Grassmannian by $G r[k]$ and denote this embedding of $\lambda^{k} M_{k n}$ by $\varphi_{k}$.

Therefore, the Mitchell-Segal filtration is in fact a filtration by algebraic varieties with $\Omega_{a l g} S U_{n}$ being the inverse limit. By a similar argument, one can show that other components of $\Omega_{a l g} U_{n}$ also are inverse limits of finite dimensional algebraic varieties.

Using Theorem 1.2, we shall prove the following

Theorem 2.3. Let $X$ be a compact irreducible subvariety of $\Omega_{a l g} U_{n}$ which is invariant under the action of $T_{\mathbb{C}} \times \mathbb{C}^{*}$. The image of $X$ under the moment map $\mu_{A P}$ is the convex hull of the images of the fixed points of the torus action which lie inside $X$.

Proof. Since $X$ is irreducible, it must be connected. Without loss of generality, we can assume that it is contained in the identity component $\Omega_{a l g} S U_{n}$. By compactness of $X$, we see that it is contained in $\lambda^{k} M_{k n}$ for some $k$, which is regarded as a subvariety of $G r[k]$.

Notice that for each $k, \lambda^{k} M_{k n}$ is invariant under the action of $T_{\mathbb{C}} \times \mathbb{C}^{*}$.

We now define an action of $T_{\mathbb{C}} \times \mathbb{C}^{*}$ (and hence of $T \times \mathbf{S}^{1}$ ) on the Grassmannian $\operatorname{Gr}[k]$. As described above, $\mathbb{C}^{k n^{2}}$ has a basis

$$
\left\{\lambda^{i} e_{j}: j=1, \cdots, n ; i=k-k n, \cdots, k-1\right\} .
$$


Clearly $T_{\mathbb{C}}$ acts on $e_{i}$ by matrix multiplication. This action induces an action on $G r[k]$. On the other hand, let $\mathbb{C}^{*}$ act on $\lambda^{i}$ by

$$
z \cdot \lambda^{i}=(z \lambda)^{i}
$$

which induces an action on $G r[k]$.

As can be easily checked, this torus action, when restricted to $X$, coincides with the torus action that comes from the loop group.

On the other hand, the Grassmannian $G r[k]$ is a Kähler manifold with the standard symplectic structure, which shall be denoted by $\omega_{k}$. It is easily seen that the torus $T \times \mathbf{S}^{1}$ preserves all these structures and a moment map $\mu_{k}: G r[k] \rightarrow \mathbf{t} \oplus \mathbb{R}$ exists.

Lemma 2.4. There is a commutative diagram:

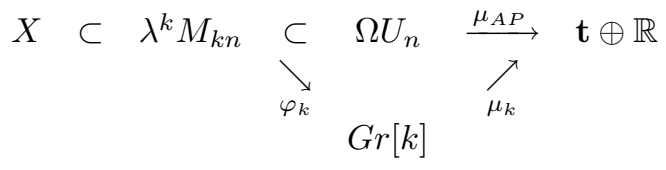

Proof. First notice that $G r[k]$ can be identified with the following set of vector subspaces:

$$
\left\{W \mid \lambda^{k} H_{+} \subset W \subset \lambda^{k-k n} H_{+} ; \quad W \text { has codimension } k n \text { in } \lambda^{k-k n} H_{+}\right\} .
$$

It is easy to check that this set is a subset of an "infinite dimensional Grassmannian" $\mathbf{G r}$ defined as the set of all closed vector subspaces $W$ of $L^{2}\left(\mathbf{S}^{1} ; \mathbb{C}^{n}\right)$ such that the orthogonal projections $p r_{ \pm}: W \rightarrow H_{ \pm}$are Fredholm and Hilbert-Schmidt operators respectively. Here $H_{-}$is the orthogonal complement of $H_{+}$in $L^{2}\left(\mathbf{S}^{1} ; \mathbb{C}^{n}\right)$. It is proved in $[\mathrm{P}-\mathrm{S}]$ that $\mathbf{G r}$ is a smooth infinite dimensional manifold such that the tangent space at each $W \in \mathbf{G r}$ is $\mathcal{I}\left(W, W^{\perp}\right)$, the Hilbert space of all HilbertSchmidt operators $W \rightarrow W^{\perp}$.

Second, the complexified torus $T_{\mathbb{C}} \times \mathbb{C}^{*}$ acts on $\mathbf{G r}$ in a similar way as its action on $G r[k]$. Moreover, $\mathbf{G r}$ has a symplectic structure $\Theta$ defined in the same fashion as the standard invariant symplectic structure on a finite dimensional Grassmannian. More precisely, for any $f$ and $g$ in $T_{W} \mathbf{G r}=\mathcal{I}\left(W, W^{\perp}\right)$, define

$$
\Theta(f, g)=-i \operatorname{Tr}\left(f^{*} g-g^{*} f\right) \text {. }
$$

Clearly, the restriction of $\Theta$ to $G r[k]$ (regarded as a subset of $\mathbf{G r}$ ) is the same as $\omega_{k}$. It is easy to check that $\Theta$ is preserved by the real torus $T \times \mathbf{S}^{1}$ and there is a moment map $\mu: \mathbf{G r} \rightarrow \mathbf{t} \oplus \mathbb{R}$.

Third, just like the embedding $\varphi$ of $M_{k n}$ into a finite dimensional Grassmannian, $\Omega U_{n}$ can be embedded into $\mathbf{G r}$ by the map

$$
\delta: \Omega U_{n} \rightarrow \mathbf{G r}: \gamma \mapsto \gamma H_{+} .
$$

Hence we have a diagram:

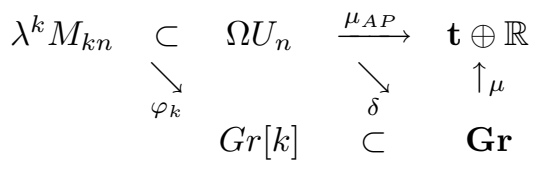

It is clear that $\mu$, when restricted to $G r[k]$, is the same as $\mu_{k}$.

We now claim that $\delta^{*}(\Theta)=\omega_{A P}$. First, it is easy to check that $\Theta$ is "invariant under multiplication of loops", in the sense that, for any $\gamma$ in $\Omega U_{n}, L_{\gamma}^{*} \Theta=\Theta$ where 
$L_{\gamma}^{*}: \mathbf{G r} \rightarrow \mathbf{G r}$ is left multiplication by $\gamma$. Together with the fact that $\omega_{A P}$ is left invariant, we see that we just need to check the claim for $T_{I} \Omega U_{n}=\Omega u_{n}$. Now, it is a straightforward matter to check that the differential $d \delta: T_{I} \Omega U_{n}=\Omega u_{n} \rightarrow$ $T_{H_{+}} \mathbf{G r}=\mathcal{I}\left(H_{+}, H_{-}\right)$at the identity loop $I \in \Omega U_{n}$ satisfies

$$
d \delta(X)=p r_{-} \circ L_{X} \in \mathcal{I}\left(H_{+}, H_{-}\right)
$$

for any $X$ in $\Omega u_{n}$. Here $p r_{-}: L^{2}\left(\mathbf{S}^{1} ; \mathbb{C}^{n}\right) \rightarrow H_{-}$is the orthogonal projection and $L_{X}$ is left multiplication by $X$.

For any $X$ and $Y$ in $\Omega u_{n}$, write them in Laurent expansions $X=\sum A_{k} e^{i k s}$ and $Y=\sum B_{m} e^{i m s}$. By straightforward calculations, one gets

$$
\omega_{A P}(X, Y)=i \sum_{k} \operatorname{Tr}\left(A_{k}^{*} B_{k}\right) .
$$

Passing to $T_{H_{+}} \mathbf{G r}=\mathcal{I}\left(H_{+}, H_{-}\right)$, let us denote the vectors $d \delta(X)=p r_{-} \circ L_{X}$ and $d \delta(Y)=p r_{-} \circ L_{Y}$ by $f_{X}$ and $f_{Y}$ respectively. We have

$$
\begin{aligned}
\Theta\left(f_{X}, f_{Y}\right)= & -i \operatorname{Tr}\left(f_{X}^{*} f_{Y}-f_{Y}^{*} f_{X}\right) \\
= & -i \sum_{j=1}^{n} \sum_{k \geq 0}\left\langle e_{j} \lambda^{k},\left(f_{X}^{*} f_{Y}-f_{Y}^{*} f_{X}\right)\left(e_{j} \lambda^{k}\right)\right\rangle_{L^{2}} \\
= & -i \sum_{k \geq 0} \sum_{j=1}^{n}\left\{\left\langle f_{X}\left(e_{j} \lambda^{k}\right), f_{Y}\left(e_{j} \lambda^{k}\right)\right\rangle_{L^{2}}\right. \\
& \left.\quad\left\langle f_{Y}\left(e_{j} \lambda^{k}\right), f_{X}\left(e_{j} \lambda^{k}\right)\right\rangle_{L^{2}}\right\}
\end{aligned}
$$

where $\lambda=e^{i s}$ and $\langle\cdot, \cdot\rangle_{L^{2}}$ is the $L^{2}$-norm. Again, by straightforward calculations, one gets

$$
\Theta\left(f_{X}, f_{y}\right)=i \sum_{k} \operatorname{Tr}\left(A_{k}^{*} B_{k}\right)
$$

which is the same as $\omega_{A P}(X, Y)$.

This claim implies that $\mu \circ \delta=\mu_{A P}$. So the above diagram is in fact commutative, from which the lemma follows.

Hence we see that the image $\mu_{A P}(X)=\mu_{k} \circ \varphi_{k}(X)$, by Theorem 1.2, coincides with the convex hull of the images of the fixed points of the torus action which lie inside $X$ under $\mu_{A P}=\mu_{k} \circ \varphi_{k}$.

The following is proved in $[\mathrm{A}-\mathrm{P}$ :

Theorem 2.5 ( $[\mathrm{A}-\mathrm{P}])$. The image of $\mu_{A P}$ on each component of $\Omega S U_{n}$ is the convex hull of the images of the fixed points of the $T \times \mathbf{S}^{1}$ action lying in that component.

The proof in [A-P] uses the group structure of $\Omega S U_{n}$.

We now show how Theorem 2.5 is in fact an easy consequence of Theorem 2.3 for which the group structure of $\Omega S U_{n}$ is not needed.

Proof of Theorem 2.5. Recall that we have

$$
\bigcup_{k \geq 0} \lambda^{k} M_{k n}=\Omega_{a l g} S U_{n} \subset \Omega U_{n} \stackrel{\mu_{A P}}{\longrightarrow} \mathbf{t} \oplus \mathbb{R} .
$$


By Theorem 2.3, we have, for each $k$,

$$
\begin{aligned}
\mu_{A P}\left(\lambda^{k} M_{k n}\right)= & \text { convex } h u l l\left\langle\mu_{A P}(m): m \text { is a fixed point of } T_{\mathbb{C}} \times \mathbb{C}^{*}\right. \text { in } \\
& \left.\lambda^{k} M_{k n}\right\rangle \\
\subset \text { convex } h u l l & \mu_{A P}(m): m \text { is a fixed point of } T_{\mathbb{C}} \times \mathbb{C}^{*} \text { in } \\
& \left.\Omega_{\text {alg }} S U_{n}\right\rangle .
\end{aligned}
$$

Hence

$$
\begin{aligned}
& \mu_{A P}\left(\Omega_{\text {alg }} S U_{n}\right)= \lim _{i \rightarrow \infty} \mu_{A P}\left(\lambda^{k} M_{k n}\right) \\
& \subset \text { convex hull }\left\langle\mu_{A P}(m): m \text { is a fixed point of } T_{\mathbb{C}} \times \mathbb{C}^{*}\right. \text { in } \\
&\left.\Omega_{\text {alg }} S U_{n}\right\rangle .
\end{aligned}
$$

On the other hand, for any $c$ in the convex hull, there exist some $m_{1}, \cdots, m_{q}$ in $\Omega_{a l g} S U_{n}$ such that $c$ is in convex hull $\left\langle\mu_{A P}\left(m_{1}\right), \cdots, \mu_{A P}\left(m_{q}\right)\right\rangle$. Clearly the points $m_{1}, \cdots, m_{q}$ must be contained in a single $\lambda^{k} M_{k n}$ for large enough $k$. Hence we have

$$
\begin{aligned}
c & \in \text { convex hull }\left\langle\mu_{A P}\left(m_{1}\right), \cdots, \mu_{A P}\left(m_{q}\right)\right\rangle \\
& \subset \text { convex hull }\left\langle\mu_{A P}(m): m \text { is a fixed point of } T_{\mathbb{C}} \times \mathbb{C}^{*} \text { in } \lambda^{k} M_{k n}\right\rangle \\
& =\mu_{A P}\left(M_{k n}\right) \\
& \subset \mu_{A P}\left(\Omega_{\text {alg }} S U_{n}\right) .
\end{aligned}
$$

Thus we have proved that

$$
\begin{aligned}
\mu_{A P}\left(\Omega_{\text {alg }} S U_{n}\right)= & \text { convex } h u l l\left\langle\mu_{A P}(m): m \text { is a fixed point of } T_{\mathbb{C}} \times \mathbb{C}^{*}\right. \text { in } \\
& \left.\Omega_{\text {alg }} S U_{n}\right\rangle .
\end{aligned}
$$

It can be easily seen from the above argument that $\mu_{A P}\left(\Omega_{a l g} S U_{n}\right)$ is a closed set.

We now claim that all $T_{\mathbb{C}} \times \mathbb{C}^{*}$-fixed points in $\Omega S U_{n}$ must be in $\Omega_{a l g} S U_{n}$. For any $\gamma(\lambda) \in \Omega S U_{n}$ which is fixed by $T_{\mathbb{C}} \times \mathbb{C}^{*}$, we have

$$
A \cdot \gamma(\lambda) \cdot A^{-1}=\gamma(\lambda)
$$

for all $A \in T$. This implies that $\gamma(\lambda)$ must be a diagonal matrix for all $\lambda \in \mathbf{S}^{1}$. For such a loop to be fixed by the $\mathbf{S}^{1}$ action, it must have the form

$$
\left(\begin{array}{cccc}
\lambda^{k_{1}} & & & \\
& \lambda^{k_{2}} & & \\
& & \ddots & \\
& & & \lambda^{k_{n}}
\end{array}\right)
$$

where each $k_{i}$ is an integer. These fixed points are easily seen to be in $\Omega_{a l g} S U_{n}$.

The above claim shows that the convex hull

$$
\text { convex hull }\left\langle\mu_{A P}(m): m \text { is a fixed point of } T_{\mathbb{C}} \times \mathbb{C}^{*} \text { in } \Omega_{\text {alg }} S U_{n}\right\rangle
$$

is the same as

convex hull $\left\langle\mu_{A P}(m): m\right.$ is a fixed point of $T_{\mathbb{C}} \times \mathbb{C}^{*}$ in $\left.\Omega S U_{n}\right\rangle$. 
It is proved in $[\mathrm{A}-\mathrm{P}](\S 5)$ that $\Omega_{a l g} S U_{n}$ is dense in $\Omega S U_{n}$. Hence we have

$$
\mu_{A P}\left(\Omega S U_{n}\right)=\mu_{A P}\left(\overline{\Omega_{a l g} S U_{n}}\right) .
$$

The theorem now follows from the fact that $\mu_{A P}\left(\Omega_{a l g} S U_{n}\right)$ is closed.

\section{ACKNOWLEDGEMENTS}

My deep gratitude goes to my thesis advisor Professor Martin Guest.

\section{REFERENCES}

[A] M.F.Atiyah, Convexity and Commuting Hamiltonians, Bull. London Math. Soc. 14 (1982), 1-15. MR 83e:53037

[A-P] M.F.Atiyah and A.N.Pressley, Convexity and Loop Groups, Progress in Mathematics 36 (1983), 33-64. MR 85e:22026

[C-S] James B. Carrell and Andrew J. Sommese, Some Topological Aspects of $\mathbb{C}^{*}$ Actions on Compact Kaehler Manifolds, Comment. Math. Helvetici 54 (1979), 567-582. MR 80m:32032

[F] T. Frankel, Fixed points and torsion on Kähler manifolds, Annals of Math. 70 (1959), 1-8. MR 24:A1730

[G-S] V. Guillemin and S. Sternberg, Convexity properties of the moment mapping, Invent. Math. 67 (1982), 491-513. MR 83m:58037

[L] W. Liu, Ph.D thesis, University of Rochester, 1998.

[M] S.A.Mitchell, The filtration of the loops on $S U(n)$ by Schubert varieties, Math. Z. 193 (1986), 374-362. MR 88d:32043

[P-S] A. Pressley and G. Segal, Loop Groups, Oxford Mathematical Monographs, Clarendon Press, 1986. MR 88i:22049

[S] G.Segal, Loop groups and Harmonic maps, Advances in homotopy theory (Cortona 1988), 153-164, London Math. Soc. Lecture Notes Ser., 139. MR 91m:58043

Department of Natural and Social Sciences, Bowling Green State University, Firelands College, One University Dr., Huron, Ohio 44839

E-mail address: nwliu@bgnet.bgsu.edu 